\title{
Verification Condition Generation for Permission Logics with Abstract Predicates and Abstraction Functions
}

\author{
Stefan Heule, Ioannis T. Kassios, Peter Müller, and Alexander J. Summers \\ ETH Zurich, Switzerland \\ stheule@ethz.ch, \\ \{ioannis. kassios, peter .mueller, alexander.summers\}@inf.ethz.ch
}

\begin{abstract}
Abstract predicates are the primary abstraction mechanism for program logics based on access permissions, such as separation logic and implicit dynamic frames. In addition to abstract predicates, it is useful to also support classical abstraction functions, for instance, to encode side-effect-free methods of the program and use them in specifications. However, combining abstract predicates and abstraction functions in a verification condition generator leads to subtle interactions, which complicate reasoning about heap modifications. Such complications may compromise soundness or cause divergence of the prover in the context of automated verification. In this paper, we present an encoding of abstract predicates and abstraction functions in the verification condition generator Boogie. Our encoding is sound and handles recursion in a way that is suitable for automatic verification using SMT solvers. It is implemented in the automatic verifier Chalice.
\end{abstract}

\section{Introduction}

Program logics based on access permissions, such as separation logic [24] and implicit dynamic frames [27] are the foundation of many program verifiers for heap-manipulating programs $[5,11,15,21,26]$. They associate an access permission with each heap location, and enforce that a method accesses a location only if it has the permission to do so. To enable modular verification, each method specification states which permissions the method requires from its caller (in its precondition) and returns to its caller (in its postcondition). Upon a call, the caller relinquishes the required permissions (we say the caller exhales the precondition) and transfers them to the callee (the callee inhales them). Conversely, when a method terminates, the method exhales its postcondition, while its caller inhales it. This technique simplifies framing; as long as a method holds on to the permission for a location (that is, does not exhale it), no other method can access that location and, thus, its value remains unchanged. When permission to a location is lost, the value recorded for that heap location should also be discarded; in the common parlance, this (outdated) information must be havoced. 


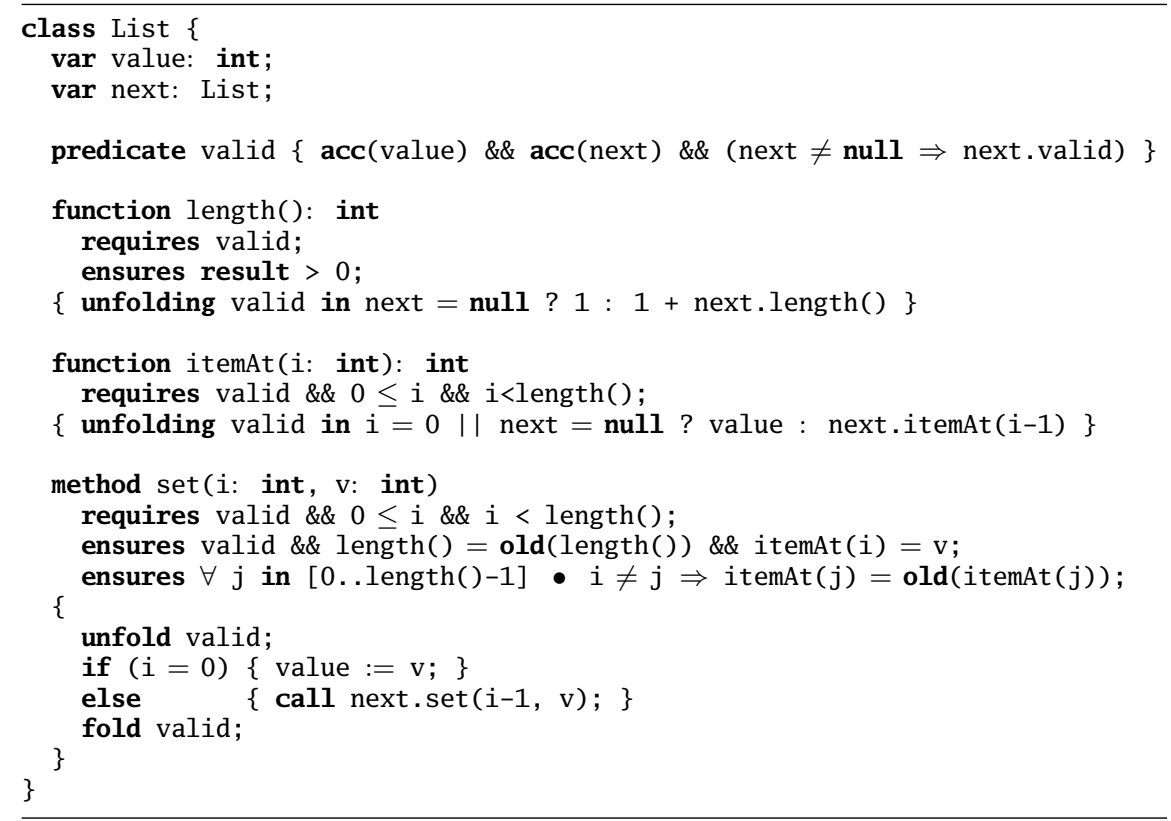

Fig. 1. A Chalice [21] implementation of a singly-linked list. Methods have preconditions (keyword requires) and postconditions (keyword ensures). In addition to regular methods, Chalice supports side-effect-free functions, which may be used in specifications. An access permission to a field $o . f$ is denoted by acc $(o . f)$, which corresponds to $o . f \mapsto \_$in separation logic. The Chalice conjunction \&\& treats permissions multiplicatively (i.e., requiring the sum of the permissions in each conjunct), similarly to the separating conjunction $*$ of separation logic. The recursive abstract predicate valid represents the memory locations of the list structure. The unfold and fold ghost statements replace a predicate by its body and vice versa. The ghost expression construct unfolding. . in is intuitively analogous to an unfold-fold block and can be used in functions and in specifications, where statements cannot occur.

Abstract Predicates. Enumerating all locations for which a method requires or returns permissions is not possible for recursive data structures. For instance, a method that traverses a linked list, such as method itemAt in Fig. 1 would require permission to access this.value, this.next, this.next.value, and so on. To solve this problem, Parkinson and Bierman [23] introduced abstract predicates. The definition of an abstract predicate declares a predicate body that may contain permissions to concrete heap locations, constraints on their values, and possibly further predicate instances. Due to this recursion, abstract predicates potentially represent permission to an unbounded number of heap locations. For instance, the abstract predicate valid in Fig. 1 represents the permissions for value, next and, if next is non-null, the permissions in next.valid. Just as with permissions to field locations, a method may require predicate instances from 
its caller. It may access a location if it possesses the corresponding permission, either directly or as part of a predicate instance. For example, method itemAt requires the predicate instance valid to get access to all locations of the list.

In this paper, we employ ghost locations to describe the predicates held for a particular object (cf. Sec. 3). Holding an instance of a predicate is represented by holding permission to its ghost location. We use the term location for both concrete field locations and predicate locations. We use the term permission to describe permissions to both kinds of locations.

Abstraction Functions. Abstraction functions [14] map the concrete representation of a data structure (say, a linked list) to an abstract value (say, a sequence). Specifications can then be expressed in terms of the abstract values, which is important for information hiding. In the form of side-effect-free inspector methods (pure methods), abstraction functions are a key ingredient of contract languages such as Eiffel, JML, Spec\#, and .NET CodeContracts, which support runtime assertion checking in addition to static verification.

Many permission logics express data abstraction via parameterised abstract predicates whose parameters represent abstracted values of a data structure and whose bodies relate the concrete representation of the data structure to these values. However, there are several advantages to supporting classical abstraction functions along with abstract predicates: (1) Most data structure implementations include side-effect-free inspector methods (or functions in Chalice) such as itemAt and length in our example. It is convenient to re-use these methods in specifications; side-effect-free methods can be encoded naturally as abstraction functions [8]. (2) Declaring abstraction functions does not affect the signatures and definitions of abstract predicates. This allows additional abstractions to be added to a library during maintenance, without changes to the predicates and re-verification of existing client code. (3) Specifications written without abstraction functions typically use logical variables for the parameters of an abstract predicate, which can then be used in postconditions to describe how the abstract value has changed. Finding witnesses for these logical variables is not supported well by SMT solvers ${ }^{1}$. By contrast, abstraction functions can be used within old expressions to refer to their pre-state evaluation, avoiding logical variables.

Abstraction function definitions must somehow be made available to the prover, so that it can relate a function application to the function's body. Since abstraction functions can be recursive, it is not possible to statically inline such definitions. In the recursive case one must also prevent the prover from unrolling the function definition infinitely often. A commonly-used approach is to use uninterpreted functions along with an axiom that relates the function to its body. In this case, the prover might select and apply such axioms infinitely often, which is known as a matching loop [9]. In our running example, unrolling the definition of the pure function $\mathrm{x}$.length() would yield the expression $\mathrm{x}$. next=null ? 1 : 1+x.next.length(), in which another call to length occurs.

${ }^{1}$ Existing tools that support rich parameterised predicates use custom reasoning engines based on symbolic execution, rather than verification condition generation. 
Verification Condition Generation Verification Condition Generation (hereafter, VCG) is a popular technique for the construction of automated verifiers $[4,7,12,17,18,20]$, in which the problem of verifying a program is encoded as a logical formula, and then handled by automatic theorem provers (typically SMT solvers). Since SMT solvers reason at a purely logical level, many problem aspects such as the program's heap state, and (for permission logics) auxiliary state to track the permissions currently held by a thread, are encoded as mathematical maps (total functions). Despite this (total) representation, a verifier can still take care that values of heap locations are only directly referred to when appropriate permissions are held. For concreteness, we present our approach in the context of Chalice, a VCG-based verifier for concurrent, imperative programs. Chalice provides only non-parameterised predicates, along with (parameterised) abstraction functions. In this setting, the primary role of predicates such as valid in Fig. 1 is to abstract over access permissions, whereas functions such as length and itemAt abstract over the contents of a data structure. Our results apply also to frameworks with parameterised predicates.

Contributions. The main contribution of this paper is an encoding of abstract predicates and abstraction functions for verification condition generators that is sound, and amenable to automation via SMT solvers.

To achieve these goals, we need solutions to the following encoding issues: (1) how to define which permissions are part of a recursive predicate instance (for example, to determine which permissions are transferred when it is exhaled), (2) how to define the values of recursive abstraction functions, and (3) how to define which heap locations the value of a recursive abstraction function depends on (for example, to determine whether a heap update affects the value of a function application).

The key insight motivating our solution is that, although each of the three issues above has, in principle, to do with a statically unbounded number of unrollings of a recursive definition, the unrolling of these definitions is typically only relevant up to the depth at which the program to be verified (including its contracts) has explicitly inspected the corresponding data structure, at either the current or an earlier program point. That is, our solution focuses on ensuring that recursively-defined information is made available for predicate and function bodies which have been syntactically observed at some program point up to the current one. Our solution employs some existing ideas, but combines and enhances them in an original way to achieve the first verification condition generator that avoids matching loops and is sound ${ }^{2}$ (the previous version of the Chalice tool was unsound, due to an incorrect encoding of abstract predicates and abstraction functions). In particular, we present a novel encoding of those permissions inside a predicate that are needed to express proof obligations.

\footnotetext{
$\overline{2}$ Some verifiers based on symbolic execution $[26,16]$ support both abstract predicates and abstraction functions, and many more support only the former $[11,15,5]$. In the equally important domain of verification condition generation, there is no solution that supports both features, is sound, and avoids matching loops.
} 
We present our solution for implicit dynamic frames [27], but it also applies to other permission logics, such as separation logic. We have implemented our solution in a new version of Chalice.

Outline. Secs. 2 and 3 present our solution informally. The details of our encoding are explained in Secs. 4 and 5, and we argue why our solution is sound in Sec. 6. We discuss related work in Sec. 7 and conclude in Sec. 8.

\section{Abstract Predicates}

In this section, we describe informally how our technique deals with the first issue described in the contributions.

\subsection{Folding and Unfolding}

Whenever a method attempts to access a heap location, the verifier needs to check whether the method has the access permission for that heap location, either directly or as part of a predicate. However, since the definitions of predicates may be recursive, a verifier cannot determine precisely which permissions are part of a predicate; since the recursion is (statically) unbounded, it is neither possible to inline the predicate's body fully, nor is it useful to let an SMT solver reason directly about recursive definitions in an unconstrained manner.

Many verifiers $[15,21,26]$ work around this problem by distinguishing between a predicate and its body. Instead of letting the SMT solver expand predicate definitions automatically, the verifier expands only specific predicate definitions at specific points in the program execution. Unfolding replaces a predicate by its body, while folding has the inverse effect. Until a predicate has been unfolded, the permissions and information implied by the predicate's body are generally not made available to the prover. We call a permission that has not been folded into a predicate instance direct; all other permissions are called folded. Accessing a field, unfolding a predicate, or exhaling a predicate all require appropriate direct permissions.

The method set in Fig. 1 illustrates these concepts. The method body first inhales its precondition; in particular, the predicate valid. After the inhale, it holds valid as a direct predicate, whereas the permissions to value and next (as well as the fields of the rest of the list) are folded. The fold statement at the end of the method is necessary to regain direct permission to valid, which gets exhaled as part of the postcondition.

Folding and unfolding transforms the problem of deciding how deeply to unroll a recursive definition to the problem of how deeply to unfold a predicate instance. Some tools provide heuristics for inferring unfold and fold operations, while others require programmers to indicate these operations through ghost statements. For our approach, it is irrelevant whether the unfold and fold operations are indicated explicitly or inferred, so long as there are specific points in 
the program execution at which the transition between a predicate and its body takes place. In our examples, we use explicit ghost statements for clarity.

It may be tempting to think that fold/unfold statements alone solve our encoding problem. Indeed these statements, explicit or inferred, protect the SMT solver from the recursion in the predicate definitions. However, a challenge that remains is when and how havocing happens. In particular, it is not useful to havoc locations immediately when the direct permission to the location is released; i.e., during a fold. The reason for this is that abstraction functions provide a way of inspecting memory locations whose permissions have been folded. If we were to havoc locations upon fold, then a function that inspects a location after is has been folded into a predicate would return an arbitrary value, which would defeat the purpose of abstraction functions. For instance, the call to itemAt in the postcondition of set would yield an unknown value, and the postcondition could not be verified.

The exhale operation now becomes complicated: it is not sufficient to havoc the locations to which direct permission is lost, but also every location that is folded under them. This set of locations is not statically known and we must find a sound way to approximate it. For example, consider that we want to exhale the predicate location this.valid, according to the definition given in Fig. 1. Not only this.valid, but every location folded under it must be havoced.

Our approach to the implementation of exhale consists of two stages. First, we havoc very aggressively: each time a predicate is exhaled, we havoc all memory locations for which the method does not retain a direct permission. This ensures soundness, but havocs too much: in fact it havocs all locations whose permissions are folded. In the second phase of our approach, we make this crude approximation more precise, by recovering an underapproximation of the location values to which we had folded permission.

The key insight mentioned in the introduction can now be made more concrete: although some information about the contents of recursively-defined predicates and functions is essential, we need concern ourselves only with those predicate instances whose bodies were unfolded earlier in the program text. Therefore, we can focus on recording detailed information for precisely these instances.

\subsection{Framing of Locations - Known-Folded Permissions}

When the verifier has knowledge of the the value of a heap location earlier in the program, it is important to preserve this information even though the permission to this location is now folded inside a predicate instance. This is a particular case of our earlier insight: it is not all folded permissions that we care about, but those (a) for which the program previously held the direct permission and (b) which are folded inside a predicate instance that has been retained up to the current program point. We call these permissions the known-folded permissions of a predicate instance. In our encoding, in addition to recording direct permissions, we record for each predicate instance those locations to which the predicate holds known-folded permission. 


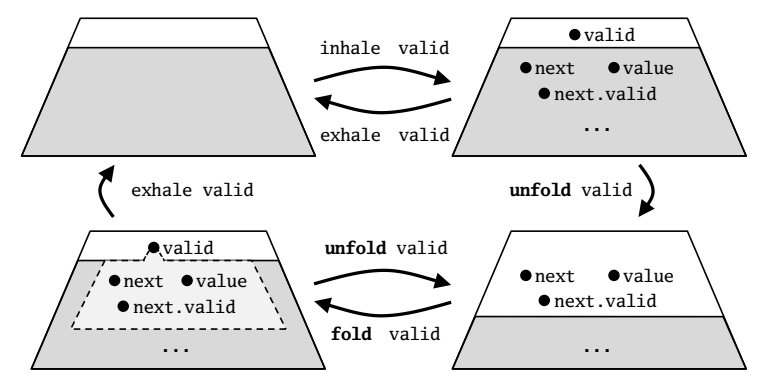

Fig. 2. Each trapezoid depicts all permissions held at a particular point in a method invocation, where the white regions contain direct permissions and the gray areas depict folded permissions. Known-folded permissions appear in the light gray region with dashed border (connected to the predicate they belong to).

Known-folded permissions provide an under-approximation of folded permissions, which (unlike the latter) can be precisely tracked in our encoding. A predicate instance contains known-folded permission to a location exactly when the direct permission to the location was folded (possibly deeply) inside the predicate instance at some earlier program point, either through a fold statement or through an unfolding expression. Because the known-folded permissions are always a subset of the folded permissions held by the current method, when exhaling a predicate it is sound to havoc only those locations for which the method holds neither direct nor known-folded permission.

Fig. 2 illustrates how our operations modify some of the possible states of the valid predicate. The upper row depicts states in which the values of fields value and next have not been observed; in the upper right trapezoid, the permissions in the body of valid are folded, but not known-folded. In the lower row the values of these fields have been observed, and permission to them is either direct or known-folded, which protects the fields from being havoced by an exhale.

As an example, consider two distinct List objects $\mathrm{x}$ and $\mathrm{y}$, for which the valid predicate is held at the beginning of the execution of the following code:

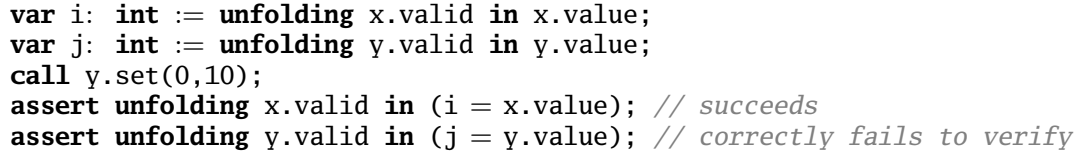

The unfolding expressions at the beginning of the code make the permissions to $\mathrm{x}$.value and y.value known-folded. The call to y.set exhales y.valid and thereby removes the permission to y.value from the known-folded permissions, since y.value is folded inside $\mathrm{x}$.value, for which the method holds known-folded permission. This makes the first assertion succeed, while the second correctly fails to verify.

If we did not havoc aggressively, the second assertion of the examples would verify, which is unsound. On the other hand, without keeping track of knownfolded permissions, the first assertion would fail to verify, which is too imprecise. 


\section{Abstraction Functions}

In this section, we explain how we handle the other two issues mentioned in the introduction, i.e., the definition and framing of abstraction functions.

\subsection{Definition of Abstraction Functions}

We encode abstraction functions as an uninterpreted function symbols together with a definitional axiom that relates the function symbol to the corresponding body. For recursive functions, this axiom might lead to a matching loop if the instantiation of the axiom is not controlled. Our approach lets the prover unroll the function definition only a statically known number of times.

A typical recursive abstraction function such as length in Fig. 1 requires permission to the locations of the data structure in its precondition (often via a predicate) and recurses on the data structure to compute its result. In particular, the body of such a function typically unfolds a predicate from its precondition before recursing on the next node in the structure via the same function (cf. length). The definitional axiom of such a function relates a function application that depends on a predicate to an expression that depends on the locations whose permissions are folded inside the predicate's body. Therefore, an instantiation of the axiom will typically only provide useful information if the verifier has observed some information about these contents. For instance, if a method does not have direct or known-folded permission to o.next and o.next.valid then it cannot observe the values of o.next and o.next.length(). Without any knowledge about these values, the definitional axiom for o.length() is not useful.

This observation leads us to tie the instantiation of function definitions to the occurrence of predicate instances which have been unfolded at some earlier point in the program code. Essentially, we again follow our key insight; it is enough to ensure that functions are unrolled to at least the depth that the corresponding predicate instances have been unfolded (at some program point up to the current one). Thus, the instantiation of axioms proceeds in lockstep with the method's traversal of the corresponding data structure.

The following example illustrates our approach. Assume that unroll is an additional method of class List. The method precondition mentions length, which allows the prover to expand the recursive definition only one level deep, which avoids a matching loop. Therefore, the prover cannot conclude from the precondition that next. next is null. However, since the method cannot observe the value of next.next at this point (the permission is folded inside valid), the information would be useless anyway. The second unfold statement unfolds the predicate instance required by the function application next.length() and, therefore, allows the prover to instantiate the definitional axiom for this application, which provides the information necessary to verify the assertion. 


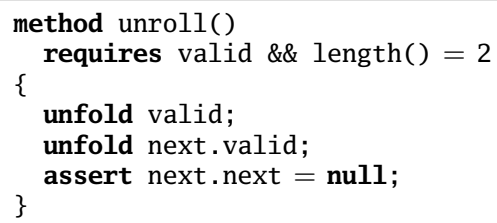

The approach outlined above avoids matching loops by controlling the instantiations of recursive definitional axioms. It supports the typical uses of abstraction functions, but does not handle recursion that is not tied to the traversal of a data structure (e.g., a factorial function). The automatic handling of such functions is beyond the scope of this paper.

\subsection{Termination of Abstraction Functions}

A common problem in allowing arbitrary recursive definitions in specifications, is the potential for introducing unsoundness to the logic, via non-well-founded recursion. For example, the definition of a function $f()=1+f()$ is inconsistent and therefore must be forbidden.

The usual approach to handling this problem is to insist on the existence of a well-founded termination measure for each recursive function definition provided. In our approach, a function passes the termination-check if the verifier can show that every recursive function call is made within the body of an unfolding expression (that is, after unfolding a predicate instance). The number of predicate instances held defines a well-founded measure because every predicate instance can be unfolded only finitely many times during the execution of a program. States at which an infinite predicate instance is held are unreachable ${ }^{3}$. Notice that the termination measure described above permits the definitions of the abstraction functions in our running example.

It is important to note that this treatment of predicates does not rule out cyclic heap structures. For instance, doubly-linked structures can be easily handled with fractional permissions, while cyclic lists can be handled (as in separation logic) using list segment predicates ${ }^{4}$.

\subsection{Framing of Abstraction Functions}

Since an SMT solver cannot unroll a recursive function definition arbitrarily deeply, it cannot use the function definitions to frame function applications, that is, to determine whether a heap update potentially affects the value of a function application or not. Therefore, an encoding of abstraction functions requires a framing axiom in addition to the definitional axiom, to express the circumstances under which the value of a function application can be framed.

Intuitively, the value of a function can be framed if none of the heap locations on which the function depends are modified. These locations are a subset of the

\footnotetext{
${ }^{3}$ A formal treatment of these issues is provided by Summers and Drossopoulou [28].

${ }^{4}$ See the online tool [1] for further examples, including a cyclic list.
} 
locations for which the function requires permission in its precondition. However, if the function requires a recursive predicate then this set cannot be determined precisely by the verifier or the SMT solver.

Similarly to existing tools such as Spec\# [4] and VeriCool [26], we handle this problem by abstracting over the locations folded inside a predicate instance, via versioning. The idea is as follows: if we can be sure that a predicate instance has been neither unfolded nor exhaled since an earlier program point, we know that all locations nested inside the predicate are unmodified. We label the predicate with the same version to identify this case.

Predicate versions are recorded as the values of predicate locations in the heap, which are treated like field locations: in particular, we retain knowledge of a predicate version so long as we hold either direct or known-folded permission to the predicate location. When we hold neither direct nor known-folded permission to such a location, it will naturally be havoced during an exhale. In addition, the version is havoced when the predicate is unfolded. Thus, our solution for function framing is closely tied to the framing of locations.

The details of our handling of functions are given in Sec. 5, but the framing axiom is, informally, as follows: two applications of the same function in two states evaluate to the same value if the receiver and all arguments of the function applications are the same and the two states agree on the values of all locations to which the function precondition requires direct permission; in particular, this includes the versions of the required predicate instances.

To illustrate our approach, consider again two distinct List objects $\mathrm{x}$ and $\mathrm{y}$ for which the valid predicate is held at the beginning in the following code:

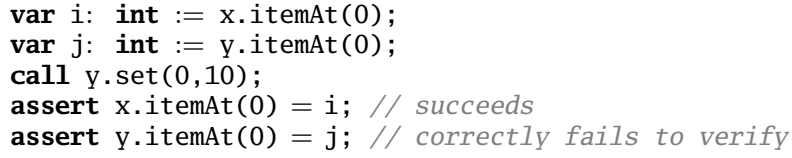

The exhale operation of the method call gives away y.valid, thereby havocing the version of that predicate. The predicate $\mathrm{x}$. valid is not affected, and keeps the same version, which allows the prover to correctly verify the first assertion, using the framing axiom. The second assertion is not necessarily true, and indeed fails to verify because the version of y.valid has changed.

\section{Encoding of Abstract Predicates}

In this and the next section, we present an encoding of our solution in the verification condition generator Boogie [18]. Verification with Boogie consists of three steps: (1) a translator translates the source program and its specification into the Boogie language, (2) Boogie computes verification conditions, and (3) an SMT solver attempts to prove the verification conditions. Here, we focus on how the translator encodes abstract predicates without giving recursive definitions or axioms to the prover. The complementary encoding of abstraction functions will be presented in the next section. 
Heaps and Permission Masks. Our encoding represents the current heap with a variable Heap, which is a map from locations to values. We use the notation $(o, l)$ to refer to the location $l$ of object $o$; its value in the map Heap is denoted by Heap $[o, l]$. Permissions are tracked using permission masks, which are sets of locations. The variable Mask stores the current mask, which represents the direct permissions held by the current method.

We also store information about predicate instances in the heap. For an abstract predicate $p$, we use (ghost) predicate locations $(o, p)$ to store a record. Such a record contains the predicate version (as an integer) along with a mask representing the known-folded permissions under the predicate instance o.p; we call this the predicate mask. We write Heap $[o, p]$.vrs to denote the version of predicate instance o.p, and Heap $[o, p]$.msk to denote the corresponding predicate mask for known-folded permissions. At the beginning of verifying a method body, we assume all predicate masks to not contain permissions, that is, to be the empty set.

For two heaps $H$ and $H^{\prime}$, and for a mask $M$, we say that $H^{\prime}$ preserves $H$ according to $M$, written $H^{\prime} \stackrel{M}{\longleftarrow} H$, if $H$ and $H^{\prime}$ agree on the location values for which direct or known-folded permission are held in the state described by $(H, M)$, and for all other predicate locations, the predicate mask in $H^{\prime}$ is empty. More precisely, $H^{\prime} \stackrel{M}{\longleftarrow} H$ if, for all locations $(o, l)$ :

1. If either $(o, l) \in M$ or $\exists\left(o^{\prime}, p^{\prime}\right) \in M$ such that $(o, l) \in H\left[o^{\prime}, p^{\prime}\right]$.msk, then: $H^{\prime}[o, l]=H[o, l]$.

2. Otherwise (when the criteria for the previous point to apply do not hold), if $l=p$ for some predicate name $p$ (i.e., $(o, l)$ is a predicate location), then: $H^{\prime}[o, p] \cdot \mathrm{msk}=\emptyset$.

An important property of our encoding is that the known-folded permissions of a predicate instance o.p include the known-folded permissions of predicates instances in the body of o.p (informally, the predicate masks record information transitively). This property is maintained as part of our encoding of a fold statement, and when evaluating an unfolding expression. Storing transitive information in this "flattened" form means we never need to recursively traverse predicate masks in our encoding.

Encoding of Exhale. As explained in Sec. 2, the exhale operation aggressively havocs the heap and preserves information only for those locations to which the method holds direct or known-folded permission after the exhale. In the encoding of exhale (see top of Fig. 3), this is reflected by introducing a fresh heap $H^{\prime}$, assuming that $H^{\prime}$ is a framed heap for the state after the exhale and then making $H^{\prime}$ the new current heap. The actual exhaling is encoded via an auxiliary operation exhale ${ }^{\prime}$, which is explained next.

The exhale' operation recursively traverses the assertion to be exhaled, asserting all logical properties, and removing the required permissions from the current mask (see Fig. 3). Exhaling a boolean expression amounts to asserting that the expression holds. Exhaling a conjunction results in exhaling the two 


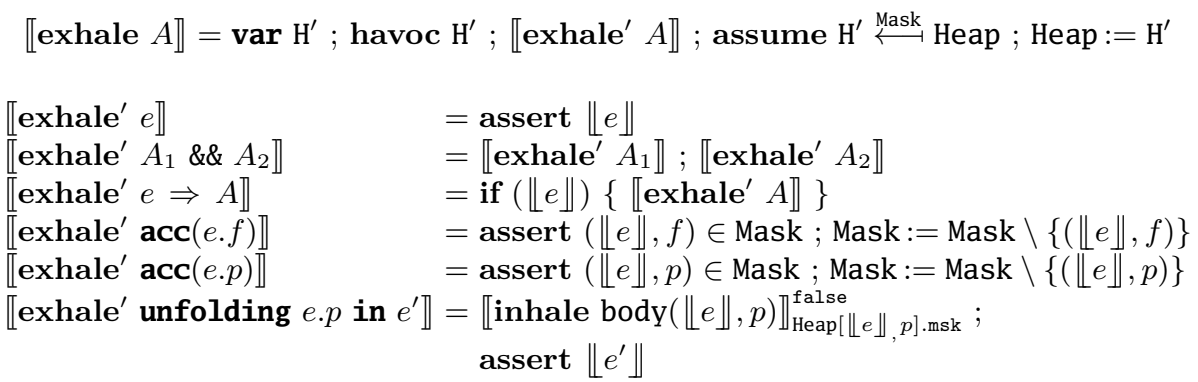

Fig. 3. Encoding of exhale. We use $A$ to denote general assertions, which may include permissions, and $e$ to denote expressions without any permissions. To emphasize the uniform treatment of field permissions and predicates, we write acc $(e . p)$ to denote the predicate instance e.p, but simply write e.p in our examples for brevity. 【_』denotes the translation of source statements to Boogie instructions. In the encoding, we treat exhale and exhale' like statements even though they cannot occur in source programs. $L_{-} \downarrow$ denotes the translation of source expressions to Boogie expressions; it is straightforward and therefore omitted. Both translation functions refer to the global variables Heap and Mask. body() yields the declared body of a predicate instance.

conjuncts sequentially; the side-effects of these exhales are accumulated. Implications are handled via if-statements in the Boogie output. Exhaling permission to a field or predicate location amounts to checking that the permission is currently held, and then removing it. Exhaling an unfolding expression is the most complicated case. First, the body of the unfolded predicate is inhaled, but using the predicate mask for the predicate instance instead of the Mask variable. This has the dual effect of assuming any logical properties from the body of the predicate, and recording the permissions encountered as known-folded (but not direct) permissions. The translation of inhale used here is described below. Finally, the body of the unfolding expression is asserted to be true.

Encoding of Inhale. The translation of inhale is given in Fig. 4. In contrast to exhale, the translation of inhale is parameterised by a mask because it sometimes operates on primary and sometimes on known-folded permissions stored in a particular predicate mask. The boolean parameter $b$ of the translation function indicates whether the inhale is of direct permissions or not. inhale traverses the assertion to be inhaled, assuming all logical properties, and adding the required permissions to the current mask. The assumption of $(\llbracket e \rrbracket, f) \notin \mathrm{M}$ in the case of inhaling field permissions encodes the fact that we cannot hold permission to the same location twice. This assumption is not made for permissions to predicate locations, since it is possible to hold the same predicate more than once $^{5}$ Additionally, when inhaling known-folded permission to a predicate e.p, all known-folded permissions from the predicate mask for e.p are added to the mask

\footnotetext{
${ }^{5}$ In this paper, this can only happen for trivial predicates without permissions, but the assumption is important once fractional permissions are employed.
} 


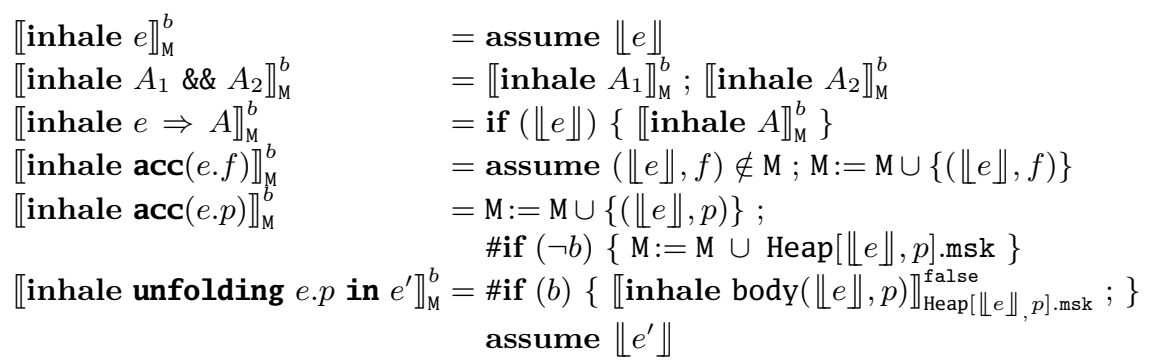

Fig. 4. Encoding of inhale. The \#if -conditionals are resolved by the translator.

being used for the inhale. In this way, we maintain the invariant that knownfolded permissions are transitively closed; that is, if $(o, p) \in$ Heap $\left[o^{\prime}, p^{\prime}\right]$.msk and $\left(o^{\prime \prime}, l^{\prime \prime}\right) \in$ Heap $[o, p]$.msk then $\left(o^{\prime \prime}, l^{\prime \prime}\right) \in$ Heap $\left[o^{\prime}, p^{\prime}\right]$.msk.

When exhaling or inhaling an unfolding expression for a predicate instance e.p, the permissions in the body of e.p become known-folded permissions. Therefore, they get inhaled into the predicate mask of e.p. In this case (which is indicated by $b$ being false), inhaling a predicate permission e.p "copies" any known-folded permissions from the inner predicate instance to the outer; that is, we flatten the known-folded permissions under e.p. Due to this flattening, an unfolding expression affects permissions only when we are inhaling direct permissions (that is, $b$ is true). If we are already inhaling known-folded permissions, any permissions folded under an inner predicate instance are already part of the known-folded masks of the outer predicates.

Encoding of Source Statements. Chalice statements are generally desugared into appropriate combinations of inhale and exhale statements. For example, a (void) method call is simply encoded with an exhale of the precondition followed by an inhale of the postcondition (see Fig. 5). The havocing of heap locations that takes place as part of the exhale takes care of invalidating any information that the called method may have changed.

fold and unfold statements do not require a general havoc of the heap, since no permissions are actually released to another method; they are simply reorganisations of permissions amongst predicate instances. Therefore, their translations use exhale'. In addition to swapping a predicate instance with its body, fold statements record known-folded permissions, and unfold statements havoc the version of the predicate instance.

We verify loops as usual by using a loop invariant and verifying the loop body independently of the surrounding code. To access locations in the loop body, the verifier requires the appropriate permissions in the loop invariant. To communicate knowledge about known-folded locations between the loop body and the surrounding context, one can use an unfolding expression in the invariant. Our encoding of unfolding then ensures that the necessary known-folded permissions are added when the loop invariant is evaluated. 


$$
\begin{aligned}
& \llbracket \text { call } \text { e.m }() \rrbracket=\llbracket \text { exhale pre }(\llbracket e \rrbracket, m) \rrbracket ; \llbracket \text { inhale post }(\llbracket e \rrbracket, m) \rrbracket_{\text {Mask }}^{\text {true }} \\
& \llbracket \text { fold } e . p \rrbracket=\llbracket \operatorname{exhale}^{\prime} \operatorname{body}(\llbracket e \rrbracket, p) \rrbracket ; \llbracket \text { inhale body }(\llbracket e \rrbracket, p) \rrbracket_{\text {Heap }[\llbracket e \Downarrow, p] \text {.msk }}^{\text {false }} ; \\
& \llbracket \text { inhale acc }(e . p) \rrbracket_{\text {Mask }}^{\text {true }} \\
& \llbracket \text { unfold } e . p \rrbracket=\llbracket \operatorname{exhale}^{\prime} \operatorname{acc}(e . p) \rrbracket ; \text { havoc Heap }[\llbracket e \rrbracket, p] . v r s \text {; } \\
& \text { Heap }[\llbracket e \rrbracket, p] . \mathrm{msk}:=\emptyset ; \llbracket \text { inhale body }(\llbracket e \rrbracket, p) \rrbracket_{\text {Mask }}^{\text {true }}
\end{aligned}
$$

Fig. 5. Encoding calls, fold, and unfold statements. pre() and post() yield the precondition and postcondition of a method with the appropriate substitutions.

\section{Encoding of Abstraction Functions}

In this section, we present our encoding of abstraction functions, building upon the handling of predicates and known-folded permissions in the previous section. The presented approach is based on uninterpreted functions and axioms, but again avoids presenting the prover with recursive axioms that can be applied in an unbounded way. This is achieved by a combination of versioning predicate instances, and careful selection of axiom triggering strategies [9].

\subsection{Function Definitional Axioms and Triggers}

Each Chalice function is represented by a corresponding uninterpreted function in the generated Boogie program, in which the heap, the receiver as well as parameters to the Chalice function are turned into explicit parameters. For example, function length() from Fig. 1 gives rise to the following uninterpreted Boogie function declaration (by convention, we prepend a \# symbol to the function name, to differentiate it from its Chalice counterpart):

function \#length(heap: HeapType, this: ref) returns (int);

In Boogie, it is standard to specify properties of an uninterpreted function via quantified axioms, e.g., defining the value of a function application, for all states. We call such an axiom (providing the definition of a function) a definitional axiom. In the common case that this axiom mentions further (recursive) function applications, the prover needs a strategy to decide when to instantiate the definitional axiom. For example, consider the "direct" translation of the Chalice definition of function length(), as a Boogie axiom (we assume here that $\mathrm{H}, \mathrm{M}$, and this range over heaps, masks, and references, respectively):

axiom $\forall \mathrm{H}, \mathrm{M}$, this $\bullet \mathrm{M}[$ this, valid $] \Longrightarrow$

\#length $(\mathrm{H}$, this $)=(\mathrm{H}[$ this, next $] \neq$ null ? $(1+\#$ length $(H, H[$ this, next $])): 1)$

This axiom states that, provided that the function's precondition holds, a function application is always equal to its body. Note that Boogie allows multiple quantified variables to be introduced together under one universal quantification. Allowing the prover to instantiate these quantifiers in arbitrary ways, would lead 
to two problems. First, the prover might instantiate the axiom with a heap and a mask that belong to different execution states. Second, since the function is recursive, the prover might instantiate its definitional axiom indefinitely in a matching loop.

To solve the first problem, our encoding introduces a boolean uninterpreted function state which takes a heap and a mask as arguments. The function application state(Heap, Mask) is assumed to yield true in our encoding every time a state is changed, for instance, after an exhale is translated (but not for the intermediate states during the translation). All axioms that quantify over a heap and mask use the state function as a premise. We write $\forall(\mathrm{H}, \mathrm{M}) \bullet \mathrm{P}(\mathrm{H}, \mathrm{M})$ to abbreviate $\forall \mathrm{H}, \mathrm{M} \bullet \operatorname{state}(\mathrm{H}, \mathrm{M}) \Rightarrow \mathrm{P}(\mathrm{H}, \mathrm{M})$.

To solve the second problem, we make use of Z3's and Boogie's facility to associate universal quantifiers with sets of syntactic triggers. A trigger set is a set of terms (the triggers), written $\{t 1, t 2, \ldots\}$, which can be associated with a $\forall$ quantification in Boogie by placing it just before the body of the quantified formula. Trigger sets do not affect the logical meaning of the formula, but prescribe a strategy for controlling the instantiation of the quantifiers introduced. The rule enforced is that the prover must have encountered (somewhere in its proof search) terms matching all of the forms $\{t 1, t 2, \ldots\}$, before a corresponding instantiation of the quantified formula can be made. Multiple trigger sets can be provided; in this case, only one set of terms need be fully matched. For example, an axiom of the form

$\forall x \bullet\{f(x), g(x)\}\{f(g(x))\} f(x)=f(g(x))$

will only allow instantiations $f(t)=f(g(t))$ to be generated only for terms $t$ such that either: terms of both forms $f(t)$ and $g(t)$ have already been encountered by the prover, or: a term of the form $f(g(t))$ has already been encountered.

In the following, we explain how we encode abstraction functions and use triggers in a way that allows the prover to obtain sufficient information from the recursive definitions of the functions, without having the possibility of entering a matching loop.

Limited and Unlimited Functions. To avoid matching loops in the definitional axioms for abstraction functions, we adopt a technique employed in other tools [19]. For each Chalice function we introduce two Boogie functions (called the limited and unlimited functions). Their logical meanings are intuitively the same, but their practical use in axioms and triggers is different. For example, for the length() function, we introduce the limited form (which we identify by adding a ' to the name) along with the original definition:

function \#length(heap: HeapType, this: ref) returns (int);

function \#length' (heap: HeapType, this: ref) returns (int);

Now, we define the definitional axiom above as follows: every occurrence of a function application which comes from the body of the function definition, is replaced by its corresponding limited function. The unlimited form of the 
function is used in the trigger set for the axiom (and is still used in the translation of source-level Chalice expressions). For example, for length we generate the following definitional axiom (all our axioms that quantify over both the heap and mask include state $(\mathrm{H}, \mathrm{M})$ as part of each trigger, but we omit this for conciseness):

axiom $\forall(H, M)$, this $\bullet\{\#$ length(H,this $)\}$ M[this, valid] $\Longrightarrow$

\#length $(\mathrm{H}$, this $)=(\mathrm{H}[$ this, next $] \neq$ null ? $(1+\#$ length' $(\mathrm{H}, \mathrm{H}[$ this, next $])): 1)$

Because the body of this axiom does not introduce any new applications of the unlimited function \#length(), an instantiation of the axiom does not give rise to any further instantiations; the potential matching loop is avoided. In order to give a meaning to the \#length' () function, the following addition axiom is used, which also does not introduce any new applications of the unlimited function \#length().

axiom $\forall(H, M)$, this $\bullet\{$ length(H,this $)\} \#$ length' $(H$, this $)=\#$ length(H, this $)$

Effectively, this allows to prover to unroll a function's definition exactly once for any given occurrence of that function in the source program.

Controlled Triggering. To implement the idea presented in Sec. 3.1 of allowing the prover to unroll a function definition when the corresponding predicate has been folded or unfolded at some point in the program, we proceed as follows. We introduce a boolean function for every predicate, to be used as a trigger for functions that depend on it. We illustrate this using the predicate valid:

function \#valid ${ }^{\text {trig }}$ (this: ref) returns (bool);

This function indicates that the corresponding predicate has been folded or unfolded in some state for the given receiver. This is introduced in our encoding by instrumenting the translation of fold e.valid and unfold e.valid with an extra assumption of \#valid ${ }^{\text {trig }}$ (e).

Additionally, we are interested in unrolling the definitional axiom for a function application with a given list of arguments only if that application for the same arguments has been mentioned somewhere in the program. Otherwise, the prover cannot learn useful information by expanding the function's definition. To this end, we add another boolean function along with an axiom:

function \#length ${ }^{\text {trig }}$ (this: ref) returns (bool)

axiom $\forall(H, M)$, this $\bullet$ \{\#length' $(H$, this $)\} \#$ length ${ }^{\text {trig }}$ (this)

We use the function application \#length ${ }^{\text {trig }}$ (e) in triggers to indicate that length has been applied to the receiver e in some state. The axiom shown encodes this meaning.

We now add an additional trigger set to our definitional axiom for length. It allows the prover to instantiate the definitional axiom in the cases described in Sec. 3.1, but still does not cause matching loops. Note that this trigger corresponds to our "key insight" in the introduction since it allows the prover to expand recursive definitions up to the depth at which the program has inspected the data structure. The resulting (and final) definitional axiom, is: 


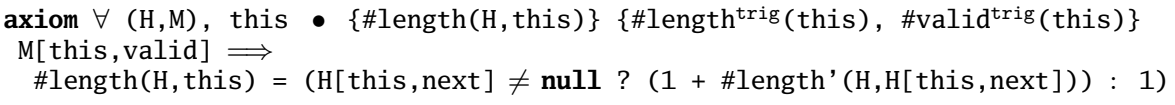

In the general case, a trigger set such as the second one above is introduced for each predicate in the precondition of the function that also gets unfolded in the body of the function before any recursive function calls that occur. If the predicate is not unfolded, or the recursive calls are not in the body of the corresponding unfolding expression, the recursion of the function is not tied to traversing the predicate over the data structure, and the additional trigger is not added.

\subsection{Function Framing Axiom}

To frame functions, we employ an additional axiom which essentially states that, if no part of the state mentioned in a function's precondition differs between two heaps, then the function's value is also the same in the two heaps. As we discussed in Sec. 3.3, we use predicate versions to abstract over the locations folded into the predicate instance, thus avoiding giving recursive predicate definitions to the prover. For example, the framing axiom for length() is as follows:

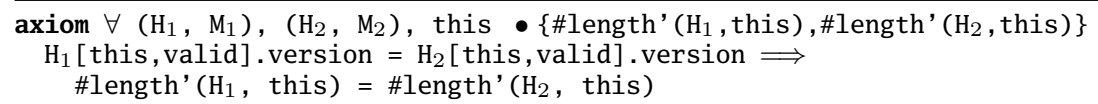

This axiom is phrased in terms of the limited function \#length', but by the axiom relating unlimited and limited functions presented in the previous subsection, it can also be used to frame unlimited functions.

Note that our use of predicate versioning is asymmetrical; if a predicate version is the same in two heaps, then we assume the contents of the predicate instance to be the same, but not vice versa. For example, unfolding and folding a predicate (without modifying any contents) will yield a new version (see Fig. 5). However, our function definitional axiom will be triggered in this case, deducing the relationship to the locations immediately contained inside the predicate instance. If these are known to be preserved, then the prover can conclude that the function value has not changed.

\section{Soundness}

In this section, we give an informal justification for the soundness of our approach. As we have explained in Sec. 3.2, the definitional axioms for the Boogie functions that we use in our encoding are consistent. The soundness arguments in this section furthermore justify (1) our approach to function framing, and (2) our approach to heap location framing. 


\subsection{Soundness of Function Framing}

To justify our approach to function framing, we use the following definition:

Definition 1 (Permission and Heap Footprints). The permission footprint of a predicate in a heap is the set of heap locations defined by recursively evaluating the predicate definition in the given heap, and collecting the locations whose permissions are required by the definition.

The permission footprint of a function in a heap is the set of heap locations defined by evaluating the function's precondition in the given heap, and collecting the locations whose permissions the precondition requires, as well as the permission footprints of all predicates the precondition requires in that evaluation.

The heap footprint of the predicate or function in a heap is the set of locationvalue pairs such that the location is in the corresponding permission footprint, and the value is the value stored in the heap at that location.

It is sufficient to observe the following:

(1) Evaluation of a function application (at runtime) reads only locations in its permission footprint. This property is enforced by a well-formedness check for each function definition, as is standard for logics supporting user-supplied definitions. In particular, a function application's value is a function of its receiver, arguments, and its heap footprint. Function bodies can also apply functions, but the checking of preconditions ensures that heap footprints for recursive applications are always subsets of the original one.

(2) Like functions, predicate definitions are checked to ensure that they read only heap locations that fall within their permission footprint. Thus, the permission and heap footprints of a predicate are fixed by the permissions folded inside of it. Since we havoc version numbers whenever a predicate is unfolded or exhaled, the version of a predicate location at two different program points can be known to be the same only if the heap footprint of the predicate is also identical at both points.

(3) Consider a situation in which our framing axiom allows the prover to equate a function value between two program points. By (1), we know that it is sufficient to know that the function's heap footprint remains the same between both points. The heap footprint is made up of locations to which explicit permission is required in the function's precondition (which the axiom requires to have the same values), and the heap footprints of those predicate locations that the function's precondition requires (which the axiom requires to have the same versions). By (2), the function value is the same in both states.

\subsection{Soundness of Heap Location Framing}

For the soundness of heap location framing, the argument depends on a precise definition of the notions of folded and known-folded locations for predicate instances. We address here the soundness of our use of predicate masks to record the known-folded permissions per predicate instance. Our encoding maintains an invariant regarding these masks, but its intuition is simple; we record and 
remember known-folded permissions until the corresponding predicate instances are lost, and make sure they are recorded in the masks of all predicate instances that enclose the locations. It is also important for our argument that we do not selectively record the known-folded permissions from a predicate's body, but always record everything that the body depends on at a time.

We need to define several notions to explain our argument. Since many of our definitions treat both types of location uniformly, we use the meta variable $l$ to range over both predicate and field location names.

\section{Definition 2 (Folded Locations).}

- In a heap $H$, a location $\left(o^{\prime}, l^{\prime}\right)$ is directly folded inside a predicate location $(o, p)$, written directFolded $\left(o, p, o^{\prime}, l^{\prime}, H\right)$, if evaluating the body of the predicate instance o.p in $H$ results in directly requiring permission to the location $\left(o^{\prime}, l^{\prime}\right)$.

- In a heap $H$, a location $\left(o^{\prime}, l^{\prime}\right)$ is folded inside a predicate location $(o, p)$, written folded $\left(o, p, o^{\prime}, l^{\prime}, H\right)$, as defined by (the least fixpoint of):

$$
\begin{aligned}
& \operatorname{folded}\left(o, p, o^{\prime}, l^{\prime}, H\right) \Leftrightarrow\left(\operatorname{directFolded}\left(o, p, o^{\prime}, l^{\prime}, H\right) \vee\right. \\
& \left.\quad \exists o^{\prime \prime}, p^{\prime \prime} .\left(\operatorname{directFolded}\left(o, p, o^{\prime \prime}, p^{\prime \prime}, H\right) \wedge \operatorname{folded}\left(o^{\prime \prime}, p^{\prime \prime}, o^{\prime}, l^{\prime}, H\right)\right)\right)
\end{aligned}
$$

We now provide the definitions which characterise the auxiliary information that our encoding records about known-folded permissions.

\section{Definition 3 (Recorded Predicate Bodies and Known-Folded Permis- sions).}

- In a heap $H$, a predicate location $(o, p)$ has its body recorded, as defined by:

$$
\begin{aligned}
& \text { bodyRecorded }(H, o, p) \Leftrightarrow\left(\forall o^{\prime}, l^{\prime} .\left(\operatorname{directFolded}\left(o, p, o^{\prime}, l^{\prime}, H\right) \Rightarrow\right.\right. \\
& \left(o^{\prime}, l^{\prime}\right) \in H[o, p] . \mathrm{msk} \wedge \\
& \left.\left(l^{\prime} \text { is a predicate location } \Rightarrow H\left[o^{\prime}, l^{\prime}\right] . \mathrm{msk} \subseteq H[o, p] . \mathrm{msk}\right)\right)
\end{aligned}
$$

- In a heap $H$, a location $\left(o^{\prime}, l^{\prime}\right)$ is known-folded inside a predicate location $(o, p)$, written knownFolded $\left(o, p, o^{\prime}, l^{\prime}, H\right)$, as defined by (the least fixpoint of):

$$
\begin{aligned}
& \text { knownFolded }\left(o, p, o^{\prime}, l^{\prime}, H\right) \Leftrightarrow \operatorname{bodyRecorded}(H, o, p) \wedge \\
& \quad\left(\operatorname{directFolded}\left(o, p, o^{\prime}, l^{\prime}, H\right) \vee\right. \\
& \left.\quad \exists o^{\prime \prime}, p^{\prime \prime} .\left(\operatorname{directFolded}\left(o, p, o^{\prime \prime}, p^{\prime \prime}, H\right) \wedge \operatorname{knownFolded}\left(o^{\prime \prime}, p^{\prime \prime}, o^{\prime}, l^{\prime}, H\right)\right)\right)
\end{aligned}
$$

Our definition of bodyRecorded requires not only that every location directly required by a predicate body is stored in the corresponding predicate mask, but also that the recorded information is transitive; any information in predicate masks for nested predicate instances must be included in the level above. Our definition of knownFolded insists on this organisation of the information in predicate masks "all the way down" - all of the predicate instances in between must also satisfy bodyRecorded. This definition of known-folded locations approximates the folded locations for a predicate instance (the definitions are similar, but knownFolded enforces extra constraints). 
One of the properties we assume about the underlying methodology, is that locations to which folded permission is held, never have their permissions also in the direct mask. This is a special case of the more-general property that permissions should never be forged/duplicated, but only transferred. Note that this is a soundness property of the underlying semantic model.

In the following we argue soundness of our particular encoding. Therefore, we use the two variables Heap and Mask to refer to the heap and mask, respectively, at a given location in the program.

Lemma 1 (Folded Locations cannot be in the Direct Mask). Before and after every Chalice program statement, it holds that

$$
\forall o, p, o^{\prime}, l^{\prime} .\left((o, p) \in \text { Mask } \wedge \operatorname{folded}\left(o, p, o^{\prime}, l^{\prime}, \text { Heap }\right)\right) \Rightarrow\left(o^{\prime}, l^{\prime}\right) \notin \text { Mask }
$$

Finally, we can state the invariant that describes how the information in our predicate masks relates to the definition of knownFolded locations above:

Theorem 1 (Predicate Mask Permissions are Known-Folded Locations). Our encoding preserves the following invariant:

$$
\begin{aligned}
& \forall o, p, o^{\prime}, l^{\prime} .\left(\left(o^{\prime}, l^{\prime}\right) \in \operatorname{Heap}[o, p] \cdot \mathrm{msk} \Rightarrow\right. \\
& \quad\left(\left((o, p) \in \operatorname{Mask} \vee \exists o^{\prime \prime}, p^{\prime \prime} .\left(\left(o^{\prime \prime}, p^{\prime \prime}\right) \in \operatorname{Mask} \wedge\right.\right.\right. \\
& \left.\left.\left.\left.\quad k n o w n F o l d e d\left(o^{\prime \prime}, p^{\prime \prime}, o, p, \text { Heap }\right)\right)\right) \wedge \text { knownFolded }\left(o, p, o^{\prime}, l^{\prime}, \text { Heap }\right)\right)\right)
\end{aligned}
$$

Proof Sketch. We show that the property is preserved across the four most relevant operations concerning known-folded permissions: folding, unfolding, inhaling, and exhaling predicate instances. For each, we assume the invariant holds beforehand, and show that it holds afterwards. To do this, we consider the cases in which the location $\left(o^{\prime}, l^{\prime}\right)$ can newly have become a member of Heap $[o, p]$.msk (in which case the consequent of the implication must also be checked), as well as the cases in which the consequent of the implication may have been falsified (in which case we must be sure that the antecedent is also now false).

Folding $o_{1} . p_{1}$ : Consider the set of locations directly required in the body of $o_{1} . p_{1}$. Permissions to all of these locations are removed from Mask and added to Heap $\left[o_{1}, p_{1}\right]$.msk. Furthermore, any of these locations which are predicate locations have their predicate masks added to Heap $\left[o_{1}, p_{1}\right] . \mathrm{msk}$ (cf. Fig. 3). In particular, these operations result in bodyRecorded(Heap, $\left.o_{1}, p_{1}\right)$ holding. Finally, $\left(o_{1}, p_{1}\right)$ is added to Mask. Since these operations only add to predicate masks, they do not falsify any previous instances of knownFolded.

Considering the invariant, the antecedent $\left(o^{\prime}, l^{\prime}\right) \in$ Heap $[o, p]$.msk can newly have been made true only in the case $o=o_{1}, p=p_{1}$ and for $\left(o^{\prime}, l^{\prime}\right)$ being one of the locations directly folded in the body of $o_{1} . p_{1}$. For all such cases, we have knownFolded $\left(o_{1}, p_{1}, o^{\prime}, l^{\prime}\right.$, Heap) as required. On the other hand, since a set of locations is removed from Mask, we must also take care that the antecedent is not falsified when $(o, p)$ is one of those locations. However, since all such locations are contained in Heap $\left[o_{1}, p_{1}\right]$.msk by the operation, the second disjunct can be shown in these cases, taking $o^{\prime \prime}=o_{1}$ and $p^{\prime \prime}=p_{1}$. 
Unfolding $o_{1} . p_{1}$ : This operation adds $\left(o_{1}, p_{1}\right)$ to Mask, and removes any information associated with the predicate instance. In particular, Heap $\left[o_{1}, p_{1}\right]$.msk is set to the empty set $\emptyset$. Since we do not add to any predicate masks, we cannot make the antecedent of the invariant true in any new cases. Since we only remove locations from the predicate mask Heap $\left[o_{1}, p_{1}\right]$.msk, the only instances of knownFolded that can be falsified by this are those concerning locations knownfolded in $\left(o_{1}, p_{1}\right)$; i.e., those $\left(o_{2}, l_{2}\right)$ for which knownFolded $\left(o_{1}, p_{1}, o_{2}, l_{2}\right.$, Heap $)$ holds (by Lemma 1 , we know that $\left(o_{1}, p_{1}\right)$ was not itself folded inside any predicate instance). Thus, considering the consequent of the invariant, the only cases we need worry about are when either $o=o_{1}$ and $p=p_{1}$ (in which case, the antecedent of the invariant is necessarily false, since we reset the predicate mask to $\emptyset$ ), or $o^{\prime \prime}=o_{1}$ and $p^{\prime \prime}=p_{1}$. In this latter case, unrolling the definition of knownFolded $\left(o^{\prime \prime}, p^{\prime \prime}, o, p\right.$, Heap) in the state before the operation, in combination with the fact that we record all direct-folded locations from the body of $o_{1} \cdot p_{1}$ in the Mask, provides sufficient information to show that the invariant still holds.

Inhaling $o_{1} \cdot p_{1}$ : This operation simply adds $\left(o_{1}, p_{1}\right)$ to Mask, which cannot falsify the consequent of the invariant, or make the antecedent newly true.

Exhaling $o_{1} \cdot p_{1}$ : This operation removes $\left(o_{1}, p_{1}\right)$ from Mask, and then generates a "global havoc", constrained by the assumption $\mathrm{H}^{\prime} \stackrel{\text { Mask }}{\longleftrightarrow}$ Heap. Let's consider the point just after this havoc operation (see Fig. 3), but before the new heap $\mathrm{H}^{\prime}$ is assigned to Heap (so that we have names for both the new and old heaps). The havoc operation means that all predicate masks $\mathrm{H}^{\prime}[o . p]$.msk are set to $\emptyset$, except in the case that either $(o, p) \in$ Mask still holds after the operation, or, for some $\left(o^{\prime \prime}, p^{\prime \prime}\right),\left(o^{\prime \prime}, p^{\prime \prime}\right) \in$ Mask $\wedge(o, p) \in$ Heap $\left[o^{\prime \prime}, p^{\prime \prime}\right]$.msk holds. In particular, consider the cases in which the antecedent of the invariant $\left(o^{\prime}, l^{\prime}\right) \in \mathrm{H}^{\prime}[o, p]$.msk could possibly hold. This would require that $\mathrm{H}^{\prime}[o, p] \cdot \mathrm{msk} \neq \emptyset$, and, since nothing is added to the predicate masks in the operation, also that $\left(o^{\prime}, l^{\prime}\right) \in$ Heap $[o, p]$.msk held. By the argument above, along with the assumption of the invariant in the state before the operation, we deduce that:

$$
\begin{aligned}
& \left(o^{\prime}, l^{\prime}\right) \in \mathrm{H}^{\prime}[o, p] . \mathrm{msk} \Rightarrow \\
& \quad\left(\left((o, p) \in \operatorname{Mask} \vee \exists o^{\prime \prime}, p^{\prime \prime} .\left(\left(o^{\prime \prime}, p^{\prime \prime}\right) \in \operatorname{Mask} \wedge\right.\right.\right. \\
& \left.\left.\left.\quad k n o w n F o l d e d\left(o^{\prime \prime}, p^{\prime \prime}, o, p, \text { Heap }\right)\right) \wedge \operatorname{knownFolded}\left(o, p, o^{\prime}, l^{\prime}, \text { Heap }\right)\right)\right)
\end{aligned}
$$

Thus, all we need to know in order to deduce that the invariant holds in the new heap $\mathrm{H}^{\prime}$ is that the occurrences of knownFolded mentioned here are still true for $\mathrm{H}^{\prime}$. This follows because, in both cases, the known-folded information is recorded under a predicate instance to which direct permission is held. By the soundness of the underlying permission logic, we know that the locations folded inside this outer predicate instance cannot be modified, and thus, that the meanings of all nested predicates are preserved. Furthermore, any predicate instances known to be folded inside this outer instance must have their entire bodies recorded in the corresponding predicate mask, and therefore, their meanings and directly folded locations remain unaffected by the global havoc. Therefore, a simple induction shows that knownFolded information is preserved in the new heap. 
Corollary 1 (Predicate Masks record only Folded Permissions). Before and after the translation of every Chalice statement, the following property holds:

$\forall o, p, o^{\prime}, l^{\prime} .\left((o, p) \in\right.$ Mask $\wedge\left(o^{\prime}, l^{\prime}\right) \in \operatorname{Heap}[o, p] . \mathrm{msk} \Rightarrow \operatorname{folded}\left(o, p, o^{\prime}, l^{\prime}\right.$, Heap $\left.)\right)$

By this corollary, which follows directly from Theorem 1, the locations framed across a global havoc are always either locations to which direct permission is held, or which are folded inside a predicate instance to which direct permission is held; in both cases, the soundness of the underlying permission handling implies that framing these location values is sound.

\section{Related Work}

The concept of abstract predicates [23] is used in both separation logic [24] and implicit dynamic frames [27]. In terms of VCG verifiers, abstraction functions are used along with abstract predicates in Chalice [20] and VeriCool [27].

The VeriCool VCG implementation handles abstract predicates and abstraction functions soundly but, unlike our solution, introduces the possibility of matching loops in the SMT solver. To assess whether matching loops are a problem in practice, we took an example from VeriCool and translated it into Chalice. We experimented with partial specifications by leaving out parts of the main loop invariant. Such verification attempts mimic and refining a program's contracts until the verification succeeds. We found that the VeriCool verification time significantly increased for failed proof attempts, from 2.3 minutes to up to one hour (at which point we terminated the verifier). In some cases, removing logically redundant parts of the specification also increased the verification time (to 16 minutes). These are typical symptoms of matching loops. Our new version of Chalice verifies the translated program in less than 20 seconds (with or without the redundant specifications) and reports failed attempts for partially specified versions even faster. Details about this experiment can be found at [1]. In particular, the site contains the code in both languages and marks the conjuncts of the VeriCool invariant according to how their removal affected the verification attempt and what the corresponding behavior of Chalice was.

The previous Chalice encoding of predicates and functions is unsound. The encoding considered only direct permissions and havoced the heap lazily, that is, when permissions are (re-)obtained. This ensures that values of fields are preserved, but also leaves invalid information in the heap, which caused the unsound behaviour. In particular, folded locations were never havoced.

Symbolic execution is an alternative technique to VCG and is used in tools such as $[5,11,15,16,26]$. Typically, symbolic execution engines use partial heaps and other more elaborate data structures for the representation of the program state. In the presence of such data structures, the problem treated in this paper is not as intricate. In particular, symbolic execution engines can iterate through their heap representation to determine folded permissions, whereas for VCG with SMT solvers, this is not possible in the presence of recursive predicates. Symbolic execution forgets heap information by chopping off the corresponding part from 
the partial heap. Framing of function values can be sufficiently handled as in VCG by predicate versioning.

The mechanism of predicate versioning typically seen in symbolic execution engines differs from ours. A version in these systems is a snapshot of the underlying heap. If a predicate is unfolded and folded back immediately, its version does not change and functions depending on that predicate are known to not have changed their value either. In contrast, our approach always changes the version number at unfold statements. However, the use of the definitional axiom allows the prover to unroll the definition one level and prove function equivalence nonetheless. In this way, we can achieve the same effect as in the predicate versioning of symbolic execution, without using the symbolic execution-specific data structures, which are unsuitable in a VCG setting. Versioning in Spec\# [4] is also similar to our predicate versioning, but more incomplete: the user must explicitly mention the functions whose return values must be preserved.

Bardou's PhD thesis [3] presents a verification technique that uses hierarchies of memory regions. To improve performance, the encoding into the Why intermediate language flattens these hierarchies into a number of separate heaps. For recursive regions, a complete flattening would result in an unbounded number of heaps. Therefore, Bardou's encoding determines statically how deeply to flatten such hierarchies, based on the access paths in a method and a fixed depth limit. Our approach requires neither such a static analysis nor a fixed limit because unfold statements determine when to expand a predicate definition, and triggers control where the prover may expand a function definition.

Madhusudan et al. [22] present a logic to express complex properties of tree structures, and a procedure that decides these efficiently. Their core idea of expanding a recursive definition a statically known number of times that depends on the program under verification is similar to ours. Compared to our work, their logic is restricted in expressiveness: it tackles only tree structures and it considers only functions with a single tree argument and a specific definition pattern.

Shape Analysis has been successfully applied in the context of three-valued logic [25], and more recently adapted to separation logic [10], with the aim of inferring the recursive structure of the current heap. The approach is typically based on a fixed set of recursive definitions, but some techniques (e.g., [13]) aim also to infer these definitions. The idea that recursive definitions need only be handled up to a certain depth is central to shape analysis, but the problem tackled is different; we do not aim at such inference, while we do support arbitrary user-defined predicates and dependent abstraction functions.

Suter et al. provide a generic approach to constructing decision procedures for recursive algebraic data types [29]. In particular, their work supports recursive abstraction functions, to allow a more abstract representation of the underlying data to be used in specifications. While their approach applies only to functional data types, they employ a notion of partial evaluation of the abstraction functions that is similar to our controlled instantiation of function definitions. It would be interesting to see if their work, as well as work on shape analyses could be adapted to our setting, perhaps to infer unfold and fold statements. 


\section{Conclusion}

In this paper, we have presented a VCG encoding technique for abstract predicates and abstraction functions. To prevent matching loops one must refrain from giving recursive definitions to the prover, even though in general the definitions of both predicates and functions can be recursive. We solve this challenge with the insight that proof obligations can typically be discharged by allowing the prover to unroll recursive definitions for the parts of the program data structures which have been observed by the program at some earlier point. Inspecting the program text allows us to encode this with the use of trigger strategies as well as the introduction of known-folded permissions.

Our encoding is, to the best of our knowledge, the only sound encoding of both features that prevents matching loops. Our comparison with the VeriCool VCG verifier shows that prevention of matching loops makes an important difference in the verification experience.

We have implemented the methodology for the more general setting of fractional permissions [6] in a new version of Chalice ${ }^{6}[2]$. We ran our implementation on the Chalice test suite of 100 interesting examples and regression tests and observed no unsoundness or incompleteness. The timings are predictable, even for examples with faulty specifications. Our tool can also be tried out online [1], where we also provide several challenging examples.

Acknowledgements. We would like to thank Jan Smans for explaining many details of the VeriCool tool to us, and Malte Schwerhoff for various discussions and for making our tool available online. We also thank Sophia Drossopoulou for early feedback on our technique, and the anonymous ECOOP reviewers for their detailed suggestions. We are also grateful to the reviewers of FM 2012 for their feedback on an earlier version of this paper, which led to a major redesign and improvement of our technique.

\section{References}

1. Chalice (online). http://boogiebox2.inf .ethz.ch:1001/tuwin/tool/chalice.

2. Chalice source code repository. http://chalice.codeplex.com.

3. R. Bardou. Verification of Pointer Programs Using Regions and Permissions. PhD thesis, Université de Paris-Sud 11, 2011.

4. M. Barnett, M. Fähndrich, K. R. M. Leino, P. Müller, W. Schulte, and H. Venter. Specification and verification: The Spec\# experience. CACM, 54(6):81-91, 2011.

5. J. Berdine, C. Calcagno, and P. W. O'Hearn. Smallfoot: Modular automatic assertion checking with separation logic. In FMCO, volume 4111 of $L N C S$, pages 115-137. Springer, 2006.

6. J. Boyland. Checking interference with fractional permissions. In $S A S$, volume 2694 of $L N C S$, pages 55-72. Springer, 2003.

\footnotetext{
${ }^{6}$ Our implementation has been successfully evaluated by the ECOOP artifact evaluation committee, who confirmed that it met their expectations.
} 
7. E. Cohen, M. Dahlweid, M. Hillebrand, D. Leinenbach, M. Moskał, T. Santen, W. Schulte, and S. Tobies. VCC: A practical system for verifying concurrent C. In TPHOLs, volume 5674 of LNCS. Springer, 2009.

8. Á. Darvas and P. Müller. Reasoning about method calls in interface specifications. JOT, 5(5):59-85, 2006.

9. D. Detlefs, G. Nelson, and J. B. Saxe. Simplify: a theorem prover for program checking. J. ACM, 52(3):365-473, 2005.

10. D. Distefano, P. W. O'Hearn, and H. Yang. A local shape analysis based on separation logic. In TACAS, volume 3920 of $L N C S$, pages 287-302. Springer, 2006.

11. D. Distefano and M. J. Parkinson. jStar: towards practical verification for Java. In OOPSLA, pages 213-226. ACM, 2008.

12. J.-C. Filliâtre and A. Paskevich. Why3 - where programs meet provers. In ESOP, volume 7792 of $L N C S$, pages 125-128. Springer, 2013.

13. B. Guo, N. Vachharajani, and D. I. August. Shape analysis with inductive recursion synthesis. In PLDI, pages 256-265. ACM, 2007.

14. C. A. R. Hoare. Proof of correctness of data representations. Acta Informatica, 1(4):271-281, 1972.

15. B. Jacobs, J. Smans, and F. Piessens. A quick tour of the VeriFast program verifier. In $A P L A S$, volume 6461 of $L N C S$, pages 304-311. Springer, 2010.

16. I. T. Kassios, P. Müller, and M. Schwerhoff. Comparing verification condition generation with symbolic execution: an experience report. In VSTTE, volume 7152 of $L N C S$, pages 196-208. Springer, 2012.

17. K. R. M. Leino. Specification and verification of object-oriented software. In Marktoberdorf International Summer School 2008, Lecture Notes, 2008.

18. K. R. M. Leino. This is Boogie 2. Working Draft. Available at http://research. microsoft.com/en-us/um/people/leino/papers.html, 2008.

19. K. R. M. Leino and R. Monahan. Reasoning about comprehensions with first-order SMT solvers. In $S A C$, pages 615-622. ACM, 2009.

20. K. R. M. Leino and P. Müller. A basis for verifying multi-threaded programs. In ESOP, volume 5502 of $L N C S$, pages 378-393. Springer, 2009.

21. K. R. M. Leino, P. Müller, and J. Smans. Verification of concurrent programs with Chalice. In FOSAD $V$, volume 5705 of $L N C S$, pages 195-222. Springer, 2009.

22. P. Madhusudan, X. Qiu, and A. Stefanescu. Recursive proofs for inductive tree data-structures. In POPL, pages 123-136. ACM, 2012.

23. M. Parkinson and G. Bierman. Separation logic and abstraction. In POPL, pages 247-258. ACM, 2005.

24. J. C. Reynolds. Separation logic: A logic for shared mutable data structures. In LICS. IEEE Computer Society Press, 2002.

25. M. Sagiv, T. Reps, and R. Wilhelm. Parametric shape analysis via 3-valued logic. In POPL, pages 105-118. ACM, 1999.

26. J. Smans, B. Jacobs, and F. Piessens. VeriCool: An automatic verifier for a concurrent object-oriented language. In FMOODS, volume 5051 of LNCS, pages 220-239. Springer, 2008.

27. J. Smans, B. Jacobs, and F. Piessens. Implicit dynamic frames: Combining dynamic frames and separation logic. In ECOOP, volume 5653 of $L N C S$, pages 148-172. Springer, 2009.

28. A. J. Summers and S. Drossopoulou. A formal semantics for isorecursive and equirecursive state abstractions. In ECOOP, LNCS. Springer, 2013.

29. P. Suter, M. Dotta, and V. Kuncak. Decision procedures for algebraic data types with abstractions. In POPL, pages 199-210. ACM, 2010. 